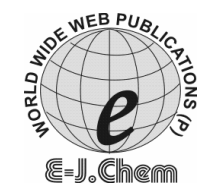

http://www.e-journals.net
CODEN ECJHAO

E-Journal of Chemistry

Vol. 3, No.2, pp 97-102, May 2006

\title{
Synthesis and Antimicrobial Activity of Newer Quinazolinones
}

\author{
J. A. PATEL, B. D. MISTRY* and K. R. DESAI** \\ *Department of Chemistry, B. K. M. Science College, Valsad-396001, \\ Affiliated with \\ **Department of Chemistry, \\ Veer Narmad South Gujarat \\ University, Surat-395007, India.
}

Received 2 January 2006; Accepted 14 April 2006

\begin{abstract}
:2-alkyl-6-bromo-3,1-benzoxazine-4-one (2) is synthesized by treating p-Bromoanthranilic acid and Acetylechoride or Benzoylchloride. Reaction of 2-alkyl-6-bromo-3,1-benzoxazine-4-one (2). with hydrazinehydrate furnish the corresponding 3-Amino-2-methyl-6-bromoquinazoline-4(3H)-one (3) which on reaction with benzaldehyde afford $\mathrm{N}, \mathrm{N}-$ arylidene derivative (4). Reaction of 4 with various diazonium salts yields 6-bromo-2-alkyl/aryl$3\{$ [phenyl(phenyldiazenyl)methylene]amino\}quinazolin-4(3H)-one .
\end{abstract}

Key words: Synthesis, quinazolidinone, antibacterial activity

\section{Introduction}

Quinazoline nucleus possessed the potent pharmacodynamic nucleus. In addition several quinazoline derivatives posses diverse biological activities Viz. anticonvulsant ${ }^{1}$, hyponotics $^{2}$, antiinflammatory ${ }^{3,4}$, diuretics antihypertensive ${ }^{5,6,7}$, antituberculor etc. quinazoline system posses variable sites at 2 and 3 positions substituted by various hetrocyclic moieties afford the potent anticonvulsant agent. Furthermore SAR study of different quinazolinone derivatives have revealed that the substitution at 3-position has significant role on hypotensive activity.

\section{Experimental}

All the melting points were determined in open capillary and are uncorrected. The purity is checked by TLC. IR Spectras were recorded in $\mathrm{KBr}$ on shimatzu F.T. - IR 8300 spectrophotometer. Analytical data were also confirmed from its H - NMR Spectra. 
The starting compound 2-alkyl/aryl-3-amino-6-bromo quinazolinone has been prepared according to known method ${ }^{8,9}$<smiles>Nc1ccccc1C(=O)O</smiles>

Anthranilic acid<smiles>CCc1cc(Br)ccc1N</smiles>

p-Bromoanthranilic acid<smiles>CCc1nc2ccc(Br)cc2c(=O)n1N</smiles>

3-Amino-2-alkyl/aryl-6bromoquinazoline-4(3h)one<smiles>[Y]c1nc2ccc(Br)cc2c(=O)n1/N=C/c1ccccc1</smiles>

3-Substituted benzalamino-2alkyl/aryl-6-bromoquinazolin-4(3H)one<smiles>[R2]c1cccc(N=NC(=Nn2c([R])nc3ccc(Br)cc3c2=O)c2ccccc2)c1</smiles>

6-bromo-2-alkyl/aryl-3-\{[phenyl(phenyldiazenyl) methylene]amino\}quinazolin-4(3H)-one

3-amino-2-alkyl/aryl-6-bromoquinazoline-4(3H)-one 3 has been prepared by known method. ${ }^{8,9}$

Synthesis of 6-bromo-2-alkyl/aryl-3\{[(1E)-phenylmethylene]amino\}quinazoline4(3H)one 4:

3-Amino-2-alkyl/aryl-7-bromoquinazoline-4(3H)-one 3(0.01 mole) and benzaldehyde $(0.01$ mole) were refluxed in absolute ethanol $(40 \mathrm{ml})$ for $3 \mathrm{hrs}$. The excess solvent was then distilled off and the resulting solid washed with water, dried and recrystallized from ethanol. 
Synthesis of 6- bromo - 2 - alkyl/aryl - 3 \{[phenyl (phenyldiazenyl) methylene] amino\} quinazoline - 4(3H) - one 5 :

Appropriate amine (0.02 Mole) in glacial Acetic acid $(2 \mathrm{ml}) \& \mathrm{HCl}(1.5 \mathrm{ml})$ was diazotised with $\mathrm{NaNO}_{2}(0.2 \mathrm{~g}$ in $2 \mathrm{ml} \mathrm{H} \mathrm{O})$ at $0-5{ }^{\circ} \mathrm{C}$. The resultant diazoniumchloride solution was added with stirring to a solution of (4) $(0.02 \mathrm{~mole})$ in pyridine. $(3 \mathrm{ml})$ in the cold. The reaction mixture. was left over night at ambient temperature. There after, it was poured into cold water $(250 \mathrm{ml})$ with stirring when a dark coloured solid separated out. It was filtered, washed, repeatedly with water containing $\mathrm{HCl}$ and finally with water and recrystallized from methanol to furnish $5 \mathrm{a}_{1}-\mathrm{a}_{16}$. (Table 1)

Table 1.Elemental analysis of synthesized compounds $\mathbf{5} \mathbf{a}_{1}-\mathbf{a}_{16}$

\begin{tabular}{|c|c|c|c|c|c|c|c|c|}
\hline \multirow[t]{2}{*}{ No. } & \multirow[t]{2}{*}{$\mathrm{R}_{1}$} & \multirow[t]{2}{*}{$\mathrm{R}_{2}$} & \multirow[t]{2}{*}{ M.F. } & \multirow{2}{*}{$\begin{array}{c}\text { Yield } \\
\%\end{array}$} & \multirow{2}{*}{$\begin{array}{l}\text { M.P. } \\
\left({ }^{\circ} \mathrm{C}\right) \\
\end{array}$} & \multicolumn{3}{|c|}{ \%Analysis Calc.(Found) } \\
\hline & & & & & & $\mathrm{C}$ & $\mathrm{H}$ & $\mathrm{N}$ \\
\hline $5 a_{1}$ & $\mathrm{CH}_{3}$ & $-\mathrm{H}$ & ${ }_{7} \mathrm{H}_{18} \mathrm{~N}_{5} \mathrm{OBr}$ & 76 & $160^{\circ} \mathrm{C}$ & $\begin{array}{c}63.79 \\
(63.20)\end{array}$ & $\begin{array}{c}3.57 \\
(4.28)\end{array}$ & $\begin{array}{c}13.78 \\
(12.40)\end{array}$ \\
\hline $5 \mathrm{a}_{2}$ & $\mathrm{CH}_{3}$ & $2-\mathrm{OCH}_{3}$ & $\mathrm{C}_{28} \mathrm{H}_{20} \mathrm{~N}_{5} \mathrm{O}_{2} \mathrm{Br}$ & 55 & $\begin{array}{l}110- \\
112^{\circ} \mathrm{C}\end{array}$ & $\begin{array}{c}62.46 \\
(62.75)\end{array}$ & $\begin{array}{c}3.74 \\
(3.94)\end{array}$ & $\begin{array}{c}13.01 \\
(13.50)\end{array}$ \\
\hline $5 \mathrm{a}_{3}$ & $\mathrm{CH}_{3}$ & $3-\mathrm{OCH}_{3}$ & $\mathrm{C}_{28} \mathrm{H}_{20} \mathrm{~N}_{5} \mathrm{O}_{2} \mathrm{Br}$ & 58 & $\begin{array}{l}113- \\
115^{\circ} \mathrm{c}\end{array}$ & $\begin{array}{c}62.46 \\
(62.75)\end{array}$ & $\begin{array}{c}3.74 \\
(3.94)\end{array}$ & $\begin{array}{c}13.01 \\
(13.50)\end{array}$ \\
\hline $5 \mathrm{a}_{4}$ & $\mathrm{CH}_{3}$ & $4-\mathrm{OCH}_{3}$ & $\mathrm{C}_{28} \mathrm{H}_{20} \mathrm{~N}_{5} \mathrm{O}_{2} \mathrm{Br}$ & 52 & $120^{\circ} \mathrm{C}$ & $\begin{array}{l}62.46 \\
(62.75)\end{array}$ & $\begin{array}{c}3.74 \\
(3.94)\end{array}$ & $\begin{array}{c}13.01 \\
(13.50)\end{array}$ \\
\hline $5 a_{5}$ & $\mathrm{CH}_{3}$ & $2-\mathrm{Br}$ & $\mathrm{C}_{27} \mathrm{H}_{17} \mathrm{~N}_{5} \mathrm{OBr}_{2}$ & 56 & $145^{\circ} \mathrm{c}$ & $\begin{array}{c}55.22 \\
(55.50)\end{array}$ & $\begin{array}{c}2.92 \\
(3.02)\end{array}$ & $\begin{array}{c}11.93 \\
(12.05)\end{array}$ \\
\hline $5 a_{6}$ & $\mathrm{CH}_{3}$ & $3-\mathrm{Br}$ & $\mathrm{C}_{27} \mathrm{H}_{17} \mathrm{~N}_{5} \mathrm{OBr}_{2}$ & 62 & $\begin{array}{l}130- \\
135^{\circ} \mathrm{c}\end{array}$ & $\begin{array}{c}55.22 \\
(55.50)\end{array}$ & $\begin{array}{c}2.92 \\
(3.02)\end{array}$ & $\begin{array}{c}11.93 \\
(12.05)\end{array}$ \\
\hline $5 a_{7}$ & $\mathrm{CH}_{3}$ & $4-\mathrm{Br}$ & $\mathrm{C}_{27} \mathrm{H}_{17} \mathrm{~N}_{5} \mathrm{OBr}_{2}$ & 80 & $155^{\circ} \mathrm{c}$ & $\begin{array}{c}55.22 \\
(55.50)\end{array}$ & $\begin{array}{c}2.92 \\
(3.02)\end{array}$ & $\begin{array}{l}11.93 \\
(12.05)\end{array}$ \\
\hline $5 \mathrm{a}_{8}$ & $\mathrm{CH}_{3}$ & $2-\mathrm{Cl}$ & $\begin{array}{c}\mathrm{C}_{27} \mathrm{H}_{17} \mathrm{~N}_{5} \mathrm{OBr} \\
\mathrm{Cl}\end{array}$ & 66 & $\begin{array}{l}136- \\
140^{\circ} \mathrm{c}\end{array}$ & $\begin{array}{c}59.74 \\
(60.03)\end{array}$ & $\begin{array}{l}3.16 \\
(3.33)\end{array}$ & $\begin{array}{c}12.90 \\
(13.15)\end{array}$ \\
\hline $5 \mathrm{a}_{9}$ & $\mathrm{CH}_{3}$ & $3-\mathrm{Cl}$ & $\begin{array}{c}\mathrm{C}_{27} \mathrm{H}_{17} \mathrm{~N}_{5} \mathrm{OBr} \\
\mathrm{Cl}\end{array}$ & 68 & $148^{\circ} \mathrm{C}$ & $\begin{array}{c}59.74 \\
(60.03)\end{array}$ & $\begin{array}{l}3.16 \\
(3.33)\end{array}$ & $\begin{array}{l}12.90 \\
(13.15)\end{array}$ \\
\hline $5 a_{10}$ & $\mathrm{CH}_{3}$ & $4-\mathrm{Cl}$ & $\begin{array}{c}\mathrm{C}_{27} \mathrm{H}_{17} \mathrm{~N}_{5} \mathrm{OBr} \\
\mathrm{Cl}\end{array}$ & 56 & $142^{\circ} \mathrm{C}$ & $\begin{array}{c}59.74 \\
(60.03)\end{array}$ & $\begin{array}{l}3.16 \\
(3.33)\end{array}$ & $\begin{array}{c}12.90 \\
(13.15)\end{array}$ \\
\hline $5 a_{11}$ & $\mathrm{CH}_{3}$ & $2-\mathrm{NO}_{2}$ & $\mathrm{C}_{27} \mathrm{H}_{17} \mathrm{~N}_{6} \mathrm{O}_{3} \mathrm{Br}$ & 68 & $\begin{array}{l}162- \\
165^{\circ} \mathrm{c}\end{array}$ & $\begin{array}{c}58.60 \\
(58.61)\end{array}$ & $\begin{array}{c}3.10 \\
(3.28)\end{array}$ & $\begin{array}{c}15.19 \\
(15.75)\end{array}$ \\
\hline $5 a_{12}$ & $\mathrm{CH}_{3}$ & $3-\mathrm{NO}_{2}$ & $\mathrm{C}_{27} \mathrm{H}_{17} \mathrm{~N}_{6} \mathrm{O}_{3} \mathrm{Br}$ & 63 & $168^{\circ} \mathrm{C}$ & $\begin{array}{c}58.60 \\
(58.61)\end{array}$ & $\begin{array}{c}3.10 \\
(3.28)\end{array}$ & $\begin{array}{c}15.19 \\
(15.75)\end{array}$ \\
\hline $5 a_{13}$ & $\mathrm{CH}_{3}$ & $4-\mathrm{NO}_{2}$ & $\mathrm{C}_{27} \mathrm{H}_{17} \mathrm{~N}_{6} \mathrm{O}_{3} \mathrm{Br}$ & 67 & $156^{\circ} \mathrm{c}$ & $\begin{array}{c}58.60 \\
(58.61)\end{array}$ & $\begin{array}{c}3.10 \\
(3.28)\end{array}$ & $\begin{array}{c}15.19 \\
(15.75)\end{array}$ \\
\hline $5 a_{14}$ & $\mathrm{CH}_{3}$ & $2-\mathrm{CH}_{3}$ & $\mathrm{C}_{28} \mathrm{H}_{20} \mathrm{~N}_{5} \mathrm{OBr}$ & 52 & $\begin{array}{l}128^{\circ} \mathrm{c} \\
\text { (d) }\end{array}$ & $\begin{array}{c}64.38 \\
(64.81)\end{array}$ & $\begin{array}{c}3.86 \\
(4.07)\end{array}$ & $\begin{array}{c}13.41 \\
(13.71)\end{array}$ \\
\hline $5 a_{15}$ & $\mathrm{CH}_{3}$ & $3-\mathrm{CH}_{3}$ & $\mathrm{C}_{28} \mathrm{H}_{20} \mathrm{~N}_{5} \mathrm{OBr}$ & 54 & $140(d)$ & $\begin{array}{c}64.38 \\
(64.81)\end{array}$ & $\begin{array}{c}3.86 \\
(4.07)\end{array}$ & $\begin{array}{c}13.41 \\
(13.71)\end{array}$ \\
\hline $5 a_{16}$ & $\begin{array}{c}\mathrm{CH}_{3} \\
\mathrm{~s} \\
\end{array}$ & $4-\mathrm{CH}_{3}$ & $\mathrm{C}_{28} \mathrm{H}_{20} \mathrm{~N}_{5} \mathrm{OBr}$ & 55 & $156(d)$ & $\begin{array}{r}64.38 \\
(64.81) \\
\end{array}$ & $\begin{array}{c}3.86 \\
(4.07) \\
\end{array}$ & $\begin{array}{c}13.41 \\
(13.71) \\
\end{array}$ \\
\hline
\end{tabular}


Table -2. Elemental analysis of synthesized compounds $\mathbf{5} \mathbf{b}_{\mathbf{1}} \mathbf{-}-\mathbf{b}_{\mathbf{1 6}}$

\begin{tabular}{|c|c|c|c|c|c|c|c|c|}
\hline \multirow[t]{2}{*}{ No. } & \multirow[t]{2}{*}{$\mathrm{R}_{1}$} & \multirow[t]{2}{*}{$\mathrm{R}_{2}$} & \multirow[t]{2}{*}{ M.F. } & \multirow{2}{*}{$\begin{array}{c}\text { Yield } \\
\%\end{array}$} & \multirow{2}{*}{$\begin{array}{l}\text { M.P. } \\
\left({ }^{\circ} \mathrm{C}\right)\end{array}$} & \multicolumn{3}{|c|}{ \%Analysis Calc.(Found) } \\
\hline & & & & & & $\mathrm{C}$ & $\mathrm{H}$ & $\mathrm{N}$ \\
\hline $5 b_{1}$ & $\mathrm{C}_{6} \mathrm{H}_{5}$ & $-\mathrm{H}$ & $\mathrm{C}_{22} \mathrm{H}_{16} \mathrm{~N}_{5} \mathrm{OBr}$ & 68 & $\begin{array}{l}179- \\
180^{\circ} \mathrm{C}\end{array}$ & $\begin{array}{c}59.21 \\
(59.20)\end{array}$ & $\begin{array}{c}3.61 \\
(3.59)\end{array}$ & $\begin{array}{c}15.69 \\
(15.40)\end{array}$ \\
\hline $5 b_{2}$ & $\mathrm{C}_{6} \mathrm{H}_{5}$ & $2-\mathrm{OCH}_{3}$ & $\mathrm{C}_{23} \mathrm{H}_{18} \mathrm{~N}_{5} \mathrm{O}_{2} \mathrm{Br}$ & 66 & $120^{\circ} \mathrm{C}$ & $\begin{array}{c}58.00 \\
(58.25)\end{array}$ & $\begin{array}{c}3.81 \\
(3.94)\end{array}$ & $\begin{array}{c}14.70 \\
(14.88)\end{array}$ \\
\hline $5 b_{3}$ & $\mathrm{C}_{6} \mathrm{H}_{5}$ & $3-\mathrm{OCH}_{3}$ & $\mathrm{C}_{23} \mathrm{H}_{18} \mathrm{~N}_{5} \mathrm{O}_{2} \mathrm{Br}$ & 65 & $124^{\circ} \mathrm{C}$ & $\begin{array}{c}58.00 \\
(58.25)\end{array}$ & $\begin{array}{c}3.81 \\
(3.94)\end{array}$ & $\begin{array}{c}14.70 \\
(14.88)\end{array}$ \\
\hline $5 b_{4}$ & $\mathrm{C}_{6} \mathrm{H}_{5}$ & $4-\mathrm{OCH}_{3}$ & $\mathrm{C}_{23} \mathrm{H}_{18} \mathrm{~N}_{5} \mathrm{O}_{2} \mathrm{Br}$ & 60 & $\begin{array}{l}130- \\
132^{\circ} \mathrm{C}\end{array}$ & $\begin{array}{c}58.00 \\
(58.25)\end{array}$ & $\begin{array}{c}3.81 \\
(3.94)\end{array}$ & $\begin{array}{c}14.70 \\
(14.88)\end{array}$ \\
\hline $5 b_{5}$ & $\mathrm{C}_{6} \mathrm{H}_{5}$ & $2-\mathrm{Br}$ & $\mathrm{C}_{22} \mathrm{H}_{15} \mathrm{~N}_{5} \mathrm{OBr}_{2}$ & 78 & $\begin{array}{l}125- \\
127^{\circ} \mathrm{C}\end{array}$ & $\begin{array}{c}50.31 \\
(50.12)\end{array}$ & $\begin{array}{c}2.88 \\
(2.75)\end{array}$ & $\begin{array}{c}13.33 \\
(13.55)\end{array}$ \\
\hline $5 b_{6}$ & $\mathrm{C}_{6} \mathrm{H}_{5}$ & $3-\mathrm{Br}$ & $\mathrm{C}_{22} \mathrm{H}_{15} \mathrm{~N}_{5} \mathrm{OBr}_{2}$ & 73 & $148^{\circ} \mathrm{C}$ & $\begin{array}{c}50.31 \\
(50.12)\end{array}$ & $\begin{array}{c}2.88 \\
(2.75)\end{array}$ & $\begin{array}{c}13.33 \\
(13.55)\end{array}$ \\
\hline $5 b_{7}$ & $\mathrm{C}_{6} \mathrm{H}_{5}$ & $4-\mathrm{Br}$ & $\mathrm{C}_{22} \mathrm{H}_{15} \mathrm{~N}_{5} \mathrm{OBr}_{2}$ & 74 & $\begin{array}{c}136- \\
137^{\circ} \mathrm{C}\end{array}$ & $\begin{array}{c}50.31 \\
(50.12)\end{array}$ & $\begin{array}{c}2.88 \\
(2.75)\end{array}$ & $\begin{array}{c}13.33 \\
(13.55)\end{array}$ \\
\hline $5 b_{8}$ & $\mathrm{C}_{6} \mathrm{H}_{5}$ & 2-Cl & $\mathrm{C}_{22} \mathrm{H}_{15} \mathrm{~N}_{5} \mathrm{OBrCl}$ & 56 & $145^{\circ} \mathrm{c}$ & $\begin{array}{c}54.96 \\
(54.87)\end{array}$ & $\begin{array}{c}3.14 \\
(3.33)\end{array}$ & $\begin{array}{c}14.57 \\
(14.76)\end{array}$ \\
\hline $5 b_{9}$ & $\mathrm{C}_{6} \mathrm{H}_{5}$ & $3-\mathrm{Cl}$ & $\mathrm{C}_{22} \mathrm{H}_{15} \mathrm{~N}_{5} \mathrm{OBrCl}$ & 52 & $\begin{array}{l}140- \\
141^{\circ} \mathrm{C}\end{array}$ & $\begin{array}{c}54.96 \\
(54.87)\end{array}$ & $\begin{array}{c}3.14 \\
(3.33)\end{array}$ & $\begin{array}{c}14.57 \\
(14.76)\end{array}$ \\
\hline $5 b_{10}$ & $\mathrm{C}_{6} \mathrm{H}_{5}$ & $4-\mathrm{Cl}$ & $\mathrm{C}_{22} \mathrm{H}_{15} \mathrm{~N}_{5} \mathrm{OBrCl}$ & 58 & $151^{\circ} \mathrm{C}$ & $\begin{array}{c}54.96 \\
(54.87)\end{array}$ & $\begin{array}{c}3.14 \\
(3.33)\end{array}$ & $\begin{array}{c}14.57 \\
(14.76)\end{array}$ \\
\hline $5 b_{11}$ & $\mathrm{C}_{6} \mathrm{H}_{5}$ & $2-\mathrm{NO}_{2}$ & $\mathrm{C}_{22} \mathrm{H}_{15} \mathrm{~N}_{6} \mathrm{O}_{3} \mathrm{Br}$ & 82 & $\begin{array}{l}170- \\
172^{\circ} \mathrm{C}\end{array}$ & $\begin{array}{c}53.78 \\
(53.91)\end{array}$ & $\begin{array}{c}3.08 \\
(3.28)\end{array}$ & $\begin{array}{c}17.11 \\
(17.35)\end{array}$ \\
\hline $5 b_{12}$ & $\mathrm{C}_{6} \mathrm{H}_{5}$ & $3-\mathrm{NO}_{2}$ & $\mathrm{C}_{22} \mathrm{H}_{15} \mathrm{~N}_{6} \mathrm{O}_{3} \mathrm{Br}$ & 76 & $165^{\circ} \mathrm{c}$ & $\begin{array}{c}53.78 \\
(53.91)\end{array}$ & $\begin{array}{c}3.08 \\
(3.28)\end{array}$ & $\begin{array}{c}17.11 \\
(17.35)\end{array}$ \\
\hline $5 b_{13}$ & $\mathrm{C}_{6} \mathrm{H}_{5}$ & $4-\mathrm{NO}_{2}$ & $\mathrm{C}_{22} \mathrm{H}_{15} \mathrm{~N}_{6} \mathrm{O}_{3} \mathrm{Br}$ & 78 & $162^{\circ} \mathrm{C}$ & $\begin{array}{c}53.78 \\
(53.91)\end{array}$ & $\begin{array}{c}3.08 \\
(3.28)\end{array}$ & $\begin{array}{c}17.11 \\
(17.35)\end{array}$ \\
\hline $5 b_{14}$ & $\mathrm{C}_{6} \mathrm{H}_{5}$ & $2-\mathrm{CH}_{3}$ & $\mathrm{C}_{23} \mathrm{H}_{18} \mathrm{~N}_{5} \mathrm{OBr}$ & 62 & $\begin{array}{c}132- \\
134^{\circ} \mathrm{c} \\
\text { (d) }\end{array}$ & $\begin{array}{c}60.01 \\
(60.23)\end{array}$ & $\begin{array}{c}3.94 \\
(4.07)\end{array}$ & $\begin{array}{c}15.21 \\
(15.43)\end{array}$ \\
\hline $5 b_{15}$ & $\mathrm{C}_{6} \mathrm{H}_{5}$ & $3-\mathrm{CH}_{3}$ & $\mathrm{C}_{23} \mathrm{H}_{18} \mathrm{~N}_{5} \mathrm{OBr}$ & 56 & $\begin{array}{c}138^{\circ} \mathrm{C} \\
\text { (d) }\end{array}$ & $\begin{array}{c}60.01 \\
(60.23)\end{array}$ & $\begin{array}{c}3.94 \\
(4.07)\end{array}$ & $\begin{array}{c}15.21 \\
(15.43)\end{array}$ \\
\hline $5 b_{16}$ & $\mathrm{C}_{6} \mathrm{H}_{5}$ & $4-\mathrm{CH}_{3}$ & $\mathrm{C}_{23} \mathrm{H}_{18} \mathrm{~N}_{5} \mathrm{OBr}$ & 58 & $\begin{array}{l}142 \\
\text { (d) }\end{array}$ & $\begin{array}{c}60.01 \\
(60.23)\end{array}$ & $\begin{array}{c}3.94 \\
(4.07)\end{array}$ & $\begin{array}{c}15.21 \\
(15.43)\end{array}$ \\
\hline
\end{tabular}

\section{Results and Discussion}

Synthesized compounds have been confirmed by elemental analysis, IR Spectra and ${ }^{1}$ HNMR Spectra.

Compound $5 \mathrm{a}_{1}$ shows IR absorption bands at $1716-1670 \mathrm{~cm}^{-1}(\mathrm{C}=0$ stretching), 1676$1670 \mathrm{~cm}^{-1}(\mathrm{C}=\mathrm{N}), 1340 \mathrm{~cm}^{-1}\left(\mathrm{Ar}-\mathrm{CH}_{3}\right), 650 \mathrm{~cm}^{-1}$ (C-Br stretching).

Compound $5 b_{1}$ shows IR absorption bands at $1734-1684 \mathrm{~cm}^{-1}(\mathrm{C}=0$ stretching) 1684 $1654 \mathrm{~cm}^{-1}(\mathrm{C}=\mathrm{N}), 652 \mathrm{~cm}^{-1}$ (C-Br stretching). 
${ }^{1} \mathrm{H}$ NMR of compound $5 \mathrm{a}_{1}: \delta 2.45\left(\mathrm{~s}, 3 \mathrm{H}, \mathrm{Ar}-\mathrm{CH}_{3}\right), 8.66\left(\mathrm{~d}, 1 \mathrm{H}, \mathrm{C}_{5}-\mathrm{H}\right), 8.25(\mathrm{~d}, 1 \mathrm{H}$, $\left.\mathrm{C}_{7}-\mathrm{H}\right), 8.12\left(\mathrm{~d}, 1 \mathrm{H}, \mathrm{C}_{8}-\mathrm{H}\right), 7.45-7.98(\mathrm{~m}, 9 \mathrm{H}, \mathrm{Ar}-\mathrm{H})$.

${ }^{1} \mathrm{H}$ NMR of compound $5 \mathrm{~b}_{1}: \delta 8.65\left(\mathrm{~d}, 1 \mathrm{H}, \mathrm{C}_{5}-\mathrm{H}\right), 8.3\left(\mathrm{~d}, 1 \mathrm{H}, \mathrm{C}_{7}-\mathrm{H}\right), 8.265\left(\mathrm{~d}, 1 \mathrm{H}, \mathrm{C}_{8}-\right.$ H), 7.4-8.2 (m, 14H, Ar-H).

\section{Antibacterial Activity}

The synthesized compounds were tested against gram positive bacteria staphylococcus aureus and Bacillus cereus and gram negative bacteria E. coli and Pseudomonas aeruginosa and a yeast candida albicans.

Table 3.Antimicrobial Activity of Newly Synthesised Compounds, Zone of Inhibition (Mm)

\begin{tabular}{|c|c|c|c|c|c|c|}
\hline $\begin{array}{l}\text { Sample } \\
\text { No. }\end{array}$ & E.coli & $\begin{array}{c}\text { Pseudomonas } \\
\text { aeruginosa }\end{array}$ & S.aureus & $\begin{array}{l}\text { Bacilus } \\
\text { cereus }\end{array}$ & Candida & $\begin{array}{c}\text { M.I.C. for } \\
\text { mycobacterium } \\
\text { tuberculosis } \\
\mu \mathrm{g} / \mathrm{ml}\end{array}$ \\
\hline $5 a_{1}$ & 8 & 9 & 8 & 9 & 00 & $>100$ \\
\hline $5 a_{2}$ & 6 & 6 & 6 & 6 & 00 & $>100$ \\
\hline $5 a_{3}$ & 8 & 9 & 6 & 6 & 00 & $>100$ \\
\hline $5 a_{4}$ & 9 & 9 & 6 & 6 & 00 & $>100$ \\
\hline $5 a_{5}$ & 10 & 8 & 11 & 6 & 00 & $>100$ \\
\hline $5 a_{6}$ & 8 & 7 & 8 & 8 & 00 & $>100$ \\
\hline $5 a_{7}$ & 7 & 7 & 8 & 8 & 00 & $>100$ \\
\hline $5 a_{8}$ & 6 & 6 & 6 & 6 & 00 & $>100$ \\
\hline $5 a_{9}$ & 6 & 6 & 6 & 6 & 00 & $>100$ \\
\hline $5 a_{10}$ & 8 & 8 & 8 & 8 & 00 & $>100$ \\
\hline $5 a_{11}$ & 6 & 9 & 8 & 8 & 00 & $>100$ \\
\hline $5 a_{12}$ & 7 & 8 & 8 & 8 & 00 & $>100$ \\
\hline $5 a_{13}$ & 9 & 8 & 8 & 7 & 00 & $>100$ \\
\hline $5 a_{14}$ & 9 & 8 & 6 & 6 & 00 & $>100$ \\
\hline $5 a_{15}$ & 8 & 9 & 6 & 6 & 00 & $>100$ \\
\hline $5 a_{16}$ & 8 & 8 & 6 & 6 & 00 & $>100$ \\
\hline $5 b_{1}$ & 9 & 8 & 9 & 9 & 00 & $>100$ \\
\hline $5 b_{2}$ & 7 & 9 & 7 & 7 & 00 & $>100$ \\
\hline $5 b_{3}$ & 8 & 8 & 6 & 6 & 00 & $>100$ \\
\hline $5 b_{4}$ & 11 & 12 & 9 & 9 & 00 & $>100$ \\
\hline $5 b_{5}$ & 8 & 7 & 8 & 8 & 00 & $>100$ \\
\hline $5 b_{6}$ & 9 & 8 & 8 & 8 & 00 & $>100$ \\
\hline $5 b_{7}$ & 8 & 8 & 6 & 6 & 00 & $>100$ \\
\hline $5 b_{8}$ & 9 & 9 & 8 & 8 & 00 & $>100$ \\
\hline $5 b_{9}$ & 8 & 8 & 9 & 9 & 00 & $>100$ \\
\hline $5 b_{10}$ & 11 & 12 & 10 & 10 & 00 & $>100$ \\
\hline $5 b_{11}$ & 8 & 8 & 9 & 9 & 00 & $>100$ \\
\hline $5 b_{12}$ & 7 & 7 & 7 & 7 & 00 & $>100$ \\
\hline $5 b_{13}$ & 9 & 8 & 8 & 9 & 00 & $>100$ \\
\hline $5 b_{14}$ & 6 & 6 & 6 & 6 & 00 & $>100$ \\
\hline $5 b_{15}$ & 6 & 6 & 6 & 7 & 00 & $>100$ \\
\hline $5 a_{16}$ & 6 & 6 & 6 & 8 & 00 & $>100$ \\
\hline
\end{tabular}


The stock solutions of compounds were prepared at a concentration of $5 \mathrm{mg} / \mathrm{ml} \&$ from stock solution the disc were prepared at a concentration of $100 \mu \mathrm{g} / \mathrm{ml}$. The testing was done on muller hinton agar plates by swabbing the agar plates with respective cultures, and placing the disc on it and incubating at $37^{\circ} \mathrm{C}$ for $24 \mathrm{hrs}$. the above results were obtained.

The compounds were also tested against mycobacterium tuberculosis at a concentration of $100 \mu \mathrm{g} / \mathrm{ml}$.

The L.J. medium was prepared from dehydrated powder following the instructions given on the dehydrated medium bottle. The compound were then added into the medium in the desired concentration and then inspisated then the prepared L.J. medium was inoculated with the pure culture of mycobacterium tuberculosis and incubated at $37^{\circ} \mathrm{C}$ for 7 weeks and then the results were read.

Standard drug tetracycline and Greseofulvin were also screened under similar conditions for comparison. Results are shown in Table 3.

\section{Acknowledgement}

We are grateful to the Department of Chemistry, B. K. M. Science College, Valsad. (Veer Narmad South Gujarat University, Surat) for providing the necessary facilities for the research work. Thanks to I.I.T. Bombay, for providing N.M.R. Spectras.

\section{References}

1 Archana, Srivastva V K, Chandra Ramesh and Ashokkumar, Indian J. Chem, 2002, 41B, 2371.

2 Buyuktimkin S, Chem Abstr, 1987, 107, 51388t.

3 Ka'si.R, Pande K, Bhalla T N, Barthwal J P, Gupta G P and Parmar S S, J. Pharma Sci., 1990, 79(4), 317.

4 Atulkumar, Singh S, Saxena A K and Shanker K, Indian J. Chem, 1988, 27B, 443.

5 Ashokkumar, Tyagi Mirdula and Srivastva V K, Indian J. Chem, 2003, 42B, 2142.

6 Pandey V K, Tulsi Sarah and Tulsi Zehra, Indian J. Chem, 2004, 3B, 180.

7 Tyagi Mirdula, Srivastva V K, Chandra Ramesh and Ashokkumar, Indian J. Chem, 2002, 41B, 2367.

8 Wasfy A A F, Indian J. Chem, 2003, 42B, 3102.

9 Yassin F A, Eissa A M F and Wasfy A A F, Indian J. Chem, 1994, 33B, 1193.

10 Dalal B Guirguis, Indian J. Chem, 2000, 39B, 264. 


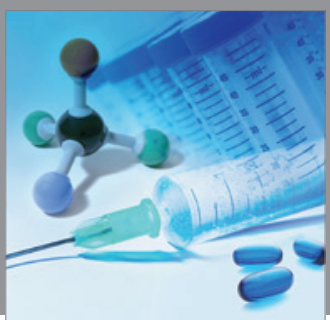

International Journal of

Medicinal Chemistry

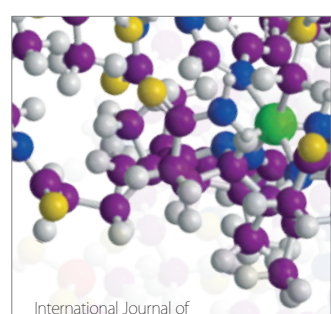

Carbohydrate Chemistry

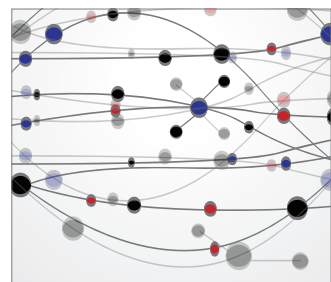

The Scientific World Journal
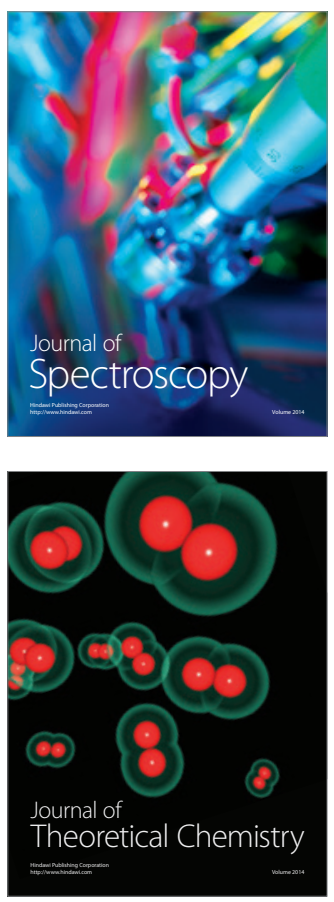
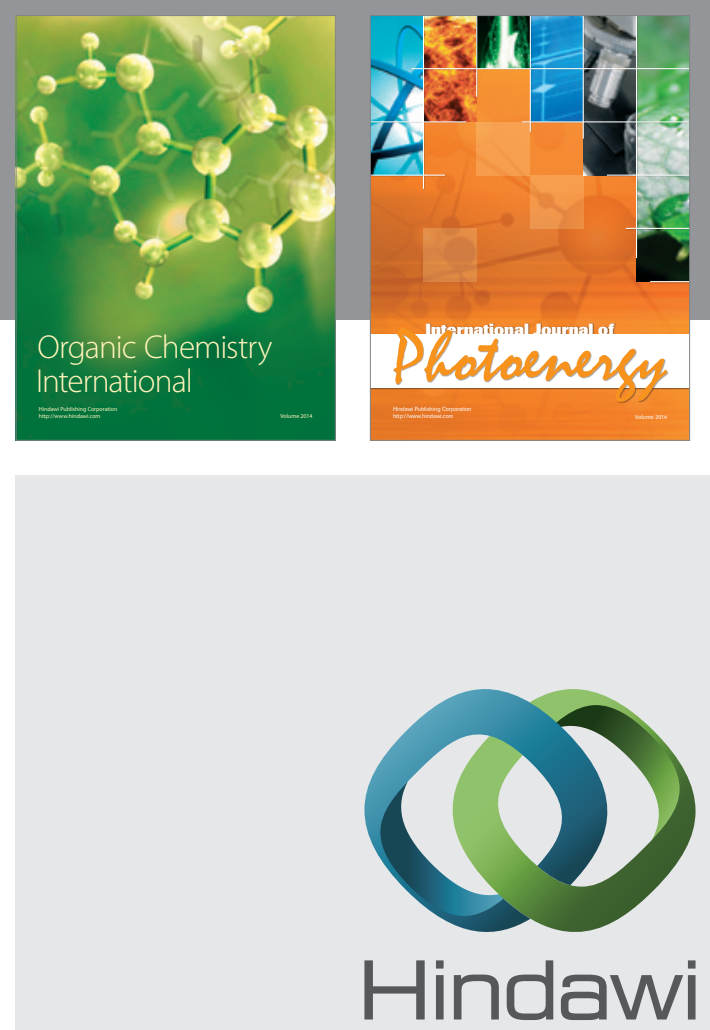

Submit your manuscripts at

http://www.hindawi.com
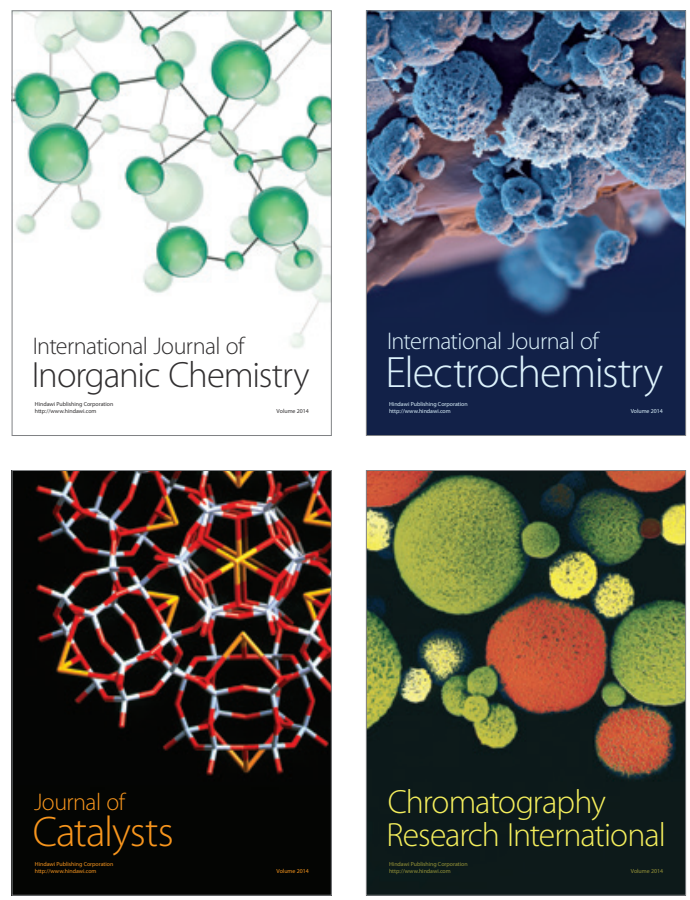
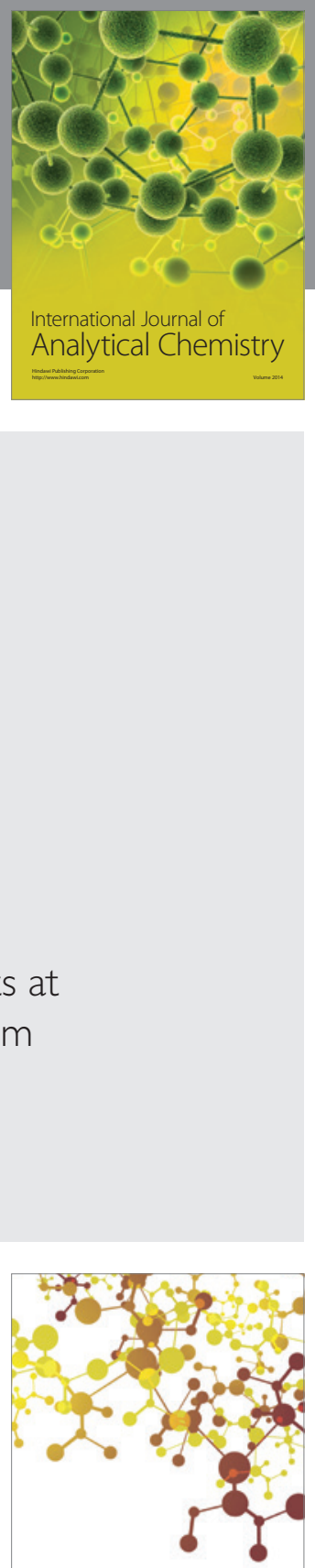

Journal of

Applied Chemistry
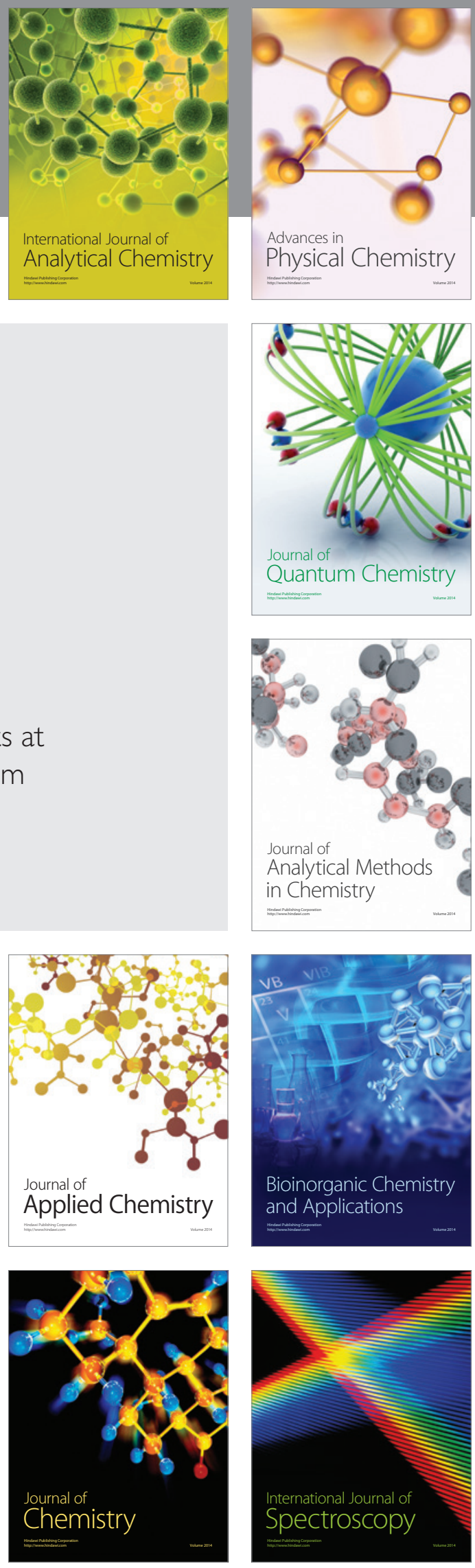